\title{
Cumplimiento de metas de lípidos en pacientes de prevención secundaria hospitalizados por medicina interna
}

\author{
Achievement of lipid goals in patients with \\ secondary prevention hospitalized in the internal \\ medicine service
}

\author{
Juan José Diaztagle, Walter Gabriel Chaves, John Jaime Sprockel, \\ Jimmy Alexander Sastoque, Jaime Andrés Nieto, Gustavo Adolfo Barreto, \\ José Ignacio HeRnÁndez • Bogotá, D.C. (Colombia)
}

\section{Resumen}

En los pacientes de prevención secundaria, el ATP III recomienda metas de lípidos estrictas (colesterol LDL $<100 \mathrm{mg} / \mathrm{dL}$ y $<70 \mathrm{mg} / \mathrm{dL}$ en pacientes de muy alto riesgo). A pesar de las recomendaciones y los múltiples beneficios demostrados de la terapia con estatinas, ésta continúa siendo subutilizada en este grupo de pacientes.

Objetivo: describir la frecuencia de cumplimiento de metas de niveles de lípidos en pacientes de prevención secundaria hospitalizados por medicina interna del Hospital San José, según criterios del ATP III.

Métodos: estudio descriptivo prospectivo, de corte transversal, realizado entre el 11 de abril y el 30 de agosto de 2011. Ingresaron pacientes mayores de 18 años con criterios de prevención secundaria. Se realizó un análisis descriptivo donde se evaluaron porcentajes, promedios y desviaciones estándares de datos demográficos, clínicos y paraclínicos. Se estableció la proporción de pacientes que cumplían metas adecuadas en el perfil lipídico según lo establecido por las guías ATP III.

Resultados: se reclutaron 121 pacientes, edad promedio $66.7 \pm 14.1$ años, predominio del sexo femenino (54.5\%). Se encontró cumplimiento de metas LDL en el 55.3\%, HDL $31.4 \%$, no HDL $51.2 \%$ y triglicéridos $52 \%$. El $57.7 \%$ recibían estatinas (todos ellos lovastatina) y 49 (40.5\%) no recibían ningún hipolipemiante.

Conclusiones: hay bajo cumplimiento según metas ATP III en los pacientes de prevención secundaria del colesterol LDL, no HDL, triglicéridos y en mayor proporción del colesterol HDL. Una gran parte de la población de estudio no recibía ningún hipolipemiante, el bajo cumplimiento puede tener diversas causas por lo que es necesario evaluarlas y buscar mecanismos para superarlas. (Acta Med Colomb 2013; 38: 54-60)

Palabras clave: términos MESH. Colesterol LDL, Dislipidemia, prevención secundaria, estatinas, Enfermedad cardiovascular.

\footnotetext{
Abstract

In secondary prevention patients, ATP III recommends strict lipid goals (LDL cholesterol $<100$ $\mathrm{mg} / \mathrm{dL}$ and $<70 \mathrm{mg} / \mathrm{dL}$ in very high-risk patients). Despite the recommendations and the multiple proven benefits of statin therapy, this continues to be underutilized in this group of patients.

Objective: to describe the frequency of compliance with lipid level goals for secondary prevention in patients hospitalized in the Internal Medical Service in San Jose Hospital, according to the ATP III criteria.

Methods: a descriptive, prospective, cross-sectional study, conducted between April 11 and August 30, 2011. Patients over 18 years with secondary prevention criteria were admitted. A descriptive analysis which evaluated percentages, means and standard deviations of demographic, clinical and
}

Dr. Juan José Diaztagle Fernández: Internista, Epidemiólogo, Magíster en Fisiología. Instructor Asistente Departamento de Medicina Interna, Fundación Universitaria de Ciencias de la Salud, Hospital de San José. Profesor Asociado Departamento de Ciencias Fisiológicas, Universidad Nacional de Colombia; Dr. Walter Gabriel Chaves Santiago: Internista. Instructor Asociado Departamento de Medicina Interna, Fundación Universitaria de Ciencias de la Salud. Jefe Servicio Medicina Interna Hospital de San José. Jefe de Posgrado Medicina Interna, Fundación Universitaria de Ciencias de la Salud; Dr. John Jaime Sprockel Díaz: Internista. Instructor Asistente Departamento de Medicina Interna Fundación Universitaria de Ciencias de la Salud, Hospital de San José; Dr. Jimmy Alexander Sastoque Torres: Internista. Fundación Universitaria de Ciencias de la Salud, Hospital Infantil de San José; Dres. Jaime Andrés Nieto Zárate y Gustavo Adolfo Barreto García: Residentes de tercer año de Medicina Interna, Fundación Universitaria de Ciencias de la Salud, Hospital de San José. Dr. José Ignacio Hernández Cruz: Internista, Cardiólogo. Profesor Emérito Departamento de Medicina Interna, Fundación Universitaria de Ciencias de la Salud, Hospital de San José. Bogotá, D.C. (Colombia).

Correspondencia. Dr. Juan José Diaztagle Fernández, Departamento de Medicina Interna, Hospital de San José. Bogotá E-mail: jjdiaztagle@fucsalud.edu.co Recibido: 19/I/2012 Aceptado: 7/III/2013 
para-clinical data was performed. The proportion of patients meeting appropriate goals in the lipid profile as defined by ATP III guidelines was established.

Results: we recruited 121 patients, mean age $66.7 \pm 14.1$ years, with female predominance $(54.5 \%)$. Achievement of LDL goals was found in 55.3\%, HDL 31.4\%, non-HDL 51.2\%and triglycerides 52\%. $57.7 \%$ received statins (lovastatin all of them) and 49 (40.5\%) did not receive any lipid-lowering drug.

Conclusions: there is poor compliance according to ATP III goals in secondary prevention patients of LDL cholesterol, non-HDL, triglycerides and increased HDL cholesterol ratio. A large proportion of the study population did not receive any lipid-lowering drug. Low compliance may have different causes; therefore it is necessary to make an evaluation and to seek ways to overcome them. (Acta

Med Colomb 2013; 38: 54-60)

Palabras clave: LDL cholesterol, dyslipidemia, secondary prevention, statins, cardiovascular disease.

\section{Introducción}

La enfermedad cardiovascular constituye la principal causa de muerte a nivel mundial al ser la responsable de casi un tercio de la mortalidad global. De ellas, la enfermedad coronaria (EC) es la causa predominante de muerte al producir aproximadamente 7.2 millones por año (1). Las causas de muerte son heterogéneas en las diferentes regiones del mundo y se ha visto que las muertes por EC han aumentado en los países de bajos y medianos ingresos (2).

En Colombia en el año 2009, la mortalidad reportada ante el DANE dio como la primera causa de muerte a las enfermedades isquémicas del corazón dando cuenta de un 14\% de todas las muertes (3). En nuestro país se calculó para el año 2007 una tasa de mortalidad atribuible a la enfermedad cardiaca isquémica de 107.3 por 100000 habitantes en personas de 45-64 años, y de 867.1 por 100000 habitantes para personas de 65 o más años (4).

Diversos estudios han demostrado que identificar y tratar agresivamente los factores de riesgo para enfermedad cardiovascular, puede significativamente impactar la morbimortalidad del paciente hasta en 18\% (5). Para la EC, estos factores incluyen: dislipidemia, tabaquismo, sedentarismo, obesidad, inadecuado control de presión arterial en hipertensos y de la glicemia en diabéticos $(6,7)$. En este contexto el colesterol de lipoproteínas de baja densidad (LDL) realiza un papel primordial en el desarrollo de la arterioesclerosis, el cual es el mecanismo fisiopatológico primario para EC. Por lo tanto, resulta muy importante un estricto control del mismo en estos pacientes $(8,9)$. También se ha establecido la importancia del colesterol no-HDL y los triglicéridos en la enfermedad cardiovascular $(10,11)$.

Por lo anterior, el tercer reporte del panel de expertos en detección, evaluación y tratamiento de hipercolesterolemia en adultos (ATP III), recomienda metas de nivel de colesterol LDL menores de $100 \mathrm{mg} / \mathrm{dL}$, de colesterol HDL mayores de $40 \mathrm{mg} / \mathrm{dL}$ en hombres y de $50 \mathrm{mg} / \mathrm{dL}$ en mujeres, de triglicéridos menores de $150 \mathrm{mg} / \mathrm{dL}$ y de colesterol no HDL menores de $130 \mathrm{mg} / \mathrm{dL}$ en pacientes con EC o equivalente coronario (diabetes mellitus, enfermedad renal crónica, enfermedad arterial periférica oclusiva o antecedente de enfermedad ce- rebrovascular) $(7,10)$.En la actualización de 2004, el ATP III definió una población de "muy alto riesgo", constituidos por pacientes con enfermedad cardiovascular y múltiples factores de riesgo mayores o pacientes con síndrome coronario agudo, en donde se establece como nueva meta el LDL menor a 70 $\mathrm{mg} / \mathrm{dL}$ (7). Estas metas también fueron propuestas por las guías americanas de prevención secundaria para pacientes con EC y enfermedad vascular aterosclerótica y las guías europeas para el manejo de las dislipidemias $(6,11)$.

Para cumplir esta meta, los medicamentos más eficaces son los inhibidores de la HMG-CoA (3-hidroxy-3-metiltglutaril coenzima A) reductasa o estatinas $(5,12)$, por lo tanto, se espera que la gran mayoría de pacientes de prevención secundaria reciban estos medicamentos a dosis suficientes para lograr las metas. Sin embargo, a pesar de la gran evidencia clínica de la utilidad de las estatinas, diversos estudios han demostrado que gran parte de los pacientes de prevención secundaria no cumple las metas establecidas por el ATP III $(5,13)$.

El contexto hospitalario brinda una oportunidad para conocer el estado actual de cada paciente en cuanto a su perfil lipídico y realizar las acciones necesarias para mejorar el cumplimiento de medidas farmacológicas y no farmacológicas, tal como es recomendado para ciertos grupos de pacientes $(6,14,15)$. En el Hospital de San José (HSJ) no se ha evaluado el cumplimiento de metas de lípidos en la población de prevención secundaria, por esta razón, el presente estudio tiene como objetivo describir la frecuencia de cumplimiento de metas terapéuticas de niveles de lípidos según lo definido por las guías ATP III, en pacientes de prevención secundaria de enfermedad cardiovascular hospitalizados por el servicio de medicina interna del HSJ de Bogotá durante el periodo comprendido entre abril 11 de 2011 y agosto 30 de 2011.

\section{Material y métodos}

Se realizó un estudio descriptivo, prospectivo, entre el 11 de abril y el 30 de agosto de 2011. Se incluyeron pacientes adultos mayores de 18 años hospitalizados por el servicio de medicina interna del HSJ de Bogotá por un tiempo mayor 
de 24 horas, que cumplían criterios para prevención secundaria de evento cardiovascular, según lo establecido por las guías ATP III: pacientes con antecedente de EC, diabetes mellitus (DM), enfermedad arterial periférica, enfermedad cerebrovascular (ECV) isquémica e insuficiencia renal crónica estadios 3, 4 o $5(10,16)$. El paciente se reclutaba si presentaba uno de los anteriores diagnósticos dentro de los antecedentes de la historia clínica de ingreso realizada por medicina interna. Se excluyeron del estudio todos aquellos pacientes con patología que genere incapacidad para comunicación con el clínico, enfermedades que modifican la expectativa de vida a corto plazo (por ejemplo, cáncer terminal), enfermedad hepática crónica avanzada o alguna otra contraindicación para el uso de estatinas.

Los datos se obtuvieron mediante la revisión de la historia clínica de ingreso realizada por el servicio de medicina interna. Se recolectaron datos sociodemográficos (edad, género, escolaridad, estrato social); medidas antropométricas (peso, talla, índice de masa corporal (IMC) y perímetro abdominal); medidas paraclínicas (niveles séricos de colesterol total, colesterol LDL, colesterol HDL y triglicéridos), variables clínicas como motivo de hospitalización (metabólico, cardiovascular, infeccioso, otros); antecedentes patológicos: hipertensión arterial, hipotiroidismo, DM tipo 1 o 2, EC, ECV isquémica, enfermedad renal crónica estadio 3 o mayor, según la clasificación KDOQI (16), enfermedad arterial periférica; antecedentes farmacológicos: consumo actual de medicamentos hipolipemiantes, antidiabéticos y antihipertensivos; y hábitos de vida (sedentarismo y tabaquismo, según definiciones de la OMS) (17).

El IMC fue calculado con la fórmula: peso/talla $(\mathrm{Kg} /$ $\mathrm{m}^{2}$ ), el perímetro abdominal fue medido con cinta métrica flexible a nivel del punto medio entre el borde inferior de las costillas y la cresta iliaca (17). Los laboratorios para evaluar cumplimiento de metas se realizaron al obtener la muestra en las primeras 24 horas de la hospitalización, con un ayuno mínimo de ocho horas. En los pacientes con antecedente de hipotiroidismo en quienes no existía dato de TSH en los últimos tres meses, se realizó medición de la misma.

La medición sérica del colesterol total, LDL, HDL y triglicéridos fue realizada por técnica de espectrofotometría de reflectancia mediante química seca a través del kit de calibrador 2 VITROS en el analizador VITROS 250 de Johnson \& Johnson. El cálculo del colesterol LDL fue realizado por el laboratorio usando la fórmula de Friedewald de Colesterol LDL $=$ Colesterol Total - Colesterol HDL- Triglicéridos $/ 5$ con nivel de triglicéridos menor de $400 \mathrm{mg} / \mathrm{dL}$. En caso contrario fue medido directamente. La medición sérica de $\mathrm{TSH}$, fue realizada por técnica de quimioluminiscencia amplificada utilizando para ello el equipo VITROS-ECI de Johnson \& Johnson.

Se definió el cumplimiento de metas de colesterol HDL, colesterol no HDL, y triglicéridos, según lo definido por el ATP III en su actualización de 2004 (7) de la siguiente forma: cumplimiento de metas de triglicéridos: menor de $150 \mathrm{mg} / \mathrm{dL}$; colesterol LDL: menor de $100 \mathrm{mg} / \mathrm{dL}$ en pacientes de alto riesgo y menor de $70 \mathrm{mg} / \mathrm{dL}$ en pacientes de muy alto riesgo; colesterol HDL: mayor de $40 \mathrm{mg} / \mathrm{dL}$ en hombres y mayor de $50 \mathrm{mg} / \mathrm{dL}$ en mujeres; y colesterol no HDL menor de $130 \mathrm{mg} / \mathrm{dL}$ o $30 \mathrm{mg} / \mathrm{dL}$ por encima de la meta de LDL si los triglicéridos son mayor de $200 \mathrm{mg} / \mathrm{dL}$. Se definió como población de "muy alto riesgo" los pacientes con enfermedad cardiovascular y una de las siguientes condiciones: 1) múltiples factores de riesgo mayores (especialmente diabetes), 2) factores de riesgo severos y pobremente controlados (especialmente la continuación del tabaquismo), 3) múltiples factores de riesgo del síndrome metabólico (especialmente triglicéridos $\geq 200 \mathrm{mg} / \mathrm{dL}$ y colesterol no-HDL $\geq 130 \mathrm{mg} / \mathrm{dL}$ con $\mathrm{HDL}<40 \mathrm{mg} / \mathrm{dL}$ ) y 4) pacientes con síndrome coronario agudo (6).

\section{Análisis estadístico}

Se diseñó una base de datos en Microsoft Excel 2007, para almacenar la información de la población en estudio. Para obtener los datos se utilizó un formato de recolección de información previamente diseñado con el total de las variables del estudio. El investigador principal se encargó de verificar la información de $20 \%$ de los formatos de recolección para garantizar la calidad de la información recolectada. El análisis estadístico se realizó con el programa STATA 10. Las variables categóricas se presentan en proporciones y las cuantitativas se resumen con promedios y desviaciones estándar. Se describe la frecuencia de cumplimiento de metas de colesterol LDL, HDL, colesterol no HDL y TG en la población total y en subgrupos de pacientes definidos según: género, antecedentes de diabetes, EC, uso de hipolipemiantes y estrato socioeconómico. Se propone realizar este análisis teniendo en cuenta que el cumplimiento de metas de manejo en estas poblaciones puede ser diferente. En casos como diabetes y EC por ejemplo, se espera que una gran parte de estos pacientes tomen estatinas y cumplan con las metas de forma más estricta. Así mismo se considera importante el antecedente de tomar estatinas, caso en el cual se espera que se cumplan mejor las metas. El protocolo fue presentado y aprobado en Comité de Investigaciones y Ética de la Facultad de Medicina de la Fundación Universitaria de Ciencias de la Salud (FUCS), HSJ, donde se consideró que dadas las características del trabajo no se requería de consentimiento informado.

\section{Resultados}

Durante el periodo comprendido entre el 11 de abril de 2011 y el 30 de agosto de 2011 ingresaron 121 pacientes al estudio. Las características demográficas de la población y las variables de interés para el estudio se anotan en la Tabla 1. De los pacientes evaluados, $66(54.5 \%)$ fueron mujeres, el promedio de edad fue de $66.7 \pm 14.1$ años. El motivo de hospitalización más frecuente fue la patología infecciosa con 66 pacientes (54.5\%) seguido por la cardiovascular 33 (27.2\%). Las enfermedades cardiovasculares más frecuen- 
Tabla 1. Características demográficas y clínicas. Pacientes 121.

\begin{tabular}{|c|c|c|c|c|c|}
\hline $\begin{array}{l}\text { Edad (promedio DE*), años } \\
\text { mínimo - máximo }\end{array}$ & $\begin{array}{c}66.7 \\
18\end{array}$ & $\begin{array}{c}(14.1) \\
96\end{array}$ & $\begin{array}{l}\text { Insuficiencia renal crónica } \neq, \mathbf{n}(\%) \\
\text { Diálisis }\end{array}$ & $\begin{array}{c}43 \\
5\end{array}$ & $\begin{array}{l}(38) \\
(4.42)\end{array}$ \\
\hline \multirow{2}{*}{$\begin{array}{l}\text { Sexo, n }(\%) \\
\text { Femenino }\end{array}$} & \multirow[b]{2}{*}{66} & \multirow[b]{2}{*}{$(54.5)$} & & & \\
\hline & & & Hipotiroidismo, n (\%) & 25 & $(20.8)$ \\
\hline \multirow{3}{*}{$\begin{array}{l}\text { Estrato socioeconómico } \dagger, \mathbf{n}(\%) \\
\text { Estrato } 1 \\
\text { Estrato } 2 \\
\text { Estrato } 3 \\
\text { Estrato } 4\end{array}$} & \multirow{3}{*}{$\begin{array}{l}2 \\
31 \\
75 \\
13\end{array}$} & \multirow{3}{*}{$\begin{array}{l}(1.6) \\
(25.7) \\
(61.9) \\
(10.7)\end{array}$} & TSH $§($ promedio DE*) & 5.1 & $(1.3)$ \\
\hline & & & mínimo - máximo & 0.62 & 21.3 \\
\hline & & & Suplencia tiroidea, $\mathbf{n}(\%)$ & 21 & $(84)$ \\
\hline \multirow{2}{*}{$\begin{array}{l}\text { Motivo de hospitalización, n (\%) } \\
\text { Metabólica } \\
\text { Infecciosa } \\
\text { Cardiovascular }\end{array}$} & \multirow[b]{2}{*}{$\begin{array}{l}22 \\
66 \\
33\end{array}$} & \multirow[b]{2}{*}{$\begin{array}{l}(18.1) \\
(54.5) \\
(27.2)\end{array}$} & Enfermedad arterial periférica, $n(\%)$ & 15 & $(12.4)$ \\
\hline & & & Uso crónico de esteroides, $n$ (\%) & 7 & $(5.79)$ \\
\hline Antecedente de enfermedad coronaria, $n(\%)$ & 19 & $(15.7)$ & $\begin{array}{l}\text { Peso (promedio DE*), } \\
\text { mínimo - máximo }\end{array}$ & $\begin{array}{c}67.4 \\
44\end{array}$ & $\begin{array}{l}(34) \\
107\end{array}$ \\
\hline $\begin{array}{l}\text { Revascularización, } \mathbf{n}(\%) \\
\text { Angioplastia } \\
\text { Stent } \\
\text { Revascularización quirúrgica }\end{array}$ & $\begin{array}{l}10 \\
3 \\
2\end{array}$ & $\begin{array}{l}(8.26) \\
(2.47) \\
(1.65)\end{array}$ & $\begin{array}{l}\text { IMC (promedio DE*), Kg/m } \mathbf{m}^{2} \\
\text { mínimo - máximo } \\
\text { Peso Normal }\end{array}$ & $\begin{array}{c}26.8 \\
19.0 \\
40\end{array}$ & $\begin{array}{c}(4.21) \\
41.8 \\
(33.0)\end{array}$ \\
\hline $\begin{array}{l}\text { Diabetes mellitus, } \mathbf{n}(\%) \\
\quad \text { Diabetes mellitus } 2\end{array}$ & 61 & $(50.4)$ & $\begin{array}{l}\text { Obesidad GI } \\
\text { Obesidad GII }\end{array}$ & $\begin{array}{c}16 \\
3\end{array}$ & $\begin{array}{c}(13.22) \\
(2.5)\end{array}$ \\
\hline \multicolumn{3}{|l|}{ Tratamiento Diabetes Mellitus, n (\%) } & Obesidad GIII & 2 & $(1.65)$ \\
\hline $\begin{array}{l}\text { Sulfonilureas } \\
\text { Metiglinidas } \\
\text { Biguanidas }\end{array}$ & $\begin{array}{c}9 \\
2 \\
26\end{array}$ & \multirow{3}{*}{$\begin{array}{l}(7.4) \\
(1.6) \\
(21.5) \\
(25.6) \\
(15.7) \\
(0.83) \\
(2.48)\end{array}$} & $\begin{array}{l}\text { Perímetro abdominal hombres (promedio DE*), cm } \\
\text { mínimo - máximo }\end{array}$ & $\begin{array}{c}93.9 \\
65\end{array}$ & $\begin{array}{c}(13.0) \\
136\end{array}$ \\
\hline $\begin{array}{l}\text { Insulina NPH } \\
\text { Insulina cristalina }\end{array}$ & $\begin{array}{l}31 \\
19\end{array}$ & & Obesidad Abdominal hombres, n (\%) & 19 & $(34.5)$ \\
\hline $\begin{array}{l}\text { Análogos de insulina } \\
\text { Otros }\end{array}$ & 1 & & Perímetro abdominal mujeres (promedio DE*), cm & 90.6 & $(12.2)$ \\
\hline \multirow{2}{*}{ Hipertensión arterial, n (\%) } & \multirow{2}{*}{102} & \multirow{2}{*}{$(84.3)$} & mínimo - máximo & & \\
\hline & & & Obesidad Abdominal mujeres, $\mathbf{n}(\%) \mathrm{cm}$ & 37 & $(56.0)$ \\
\hline \multirow{4}{*}{$\begin{array}{l}\text { Tratamiento antihipertensivo, } \mathbf{n}(\%) \\
\text { Inhibidores de la ECA } \\
\text { Antagonistas de los receptores de Angiotensina } 2 \\
\text { Diuréticos } \\
\text { Betabloqueadores } \\
\text { Alfabloqueadores } \\
\text { Bloqueadores de canales de calcio } \\
\text { Alfa agonistas centrales } \\
\text { Inhibidores directo de renina }\end{array}$} & \multirow{4}{*}{$\begin{array}{c}45 \\
25 \\
46 \\
28 \\
2 \\
25 \\
4 \\
1\end{array}$} & \multirow{4}{*}{$\begin{array}{c}(37.1) \\
(20.7) \\
(38.0) \\
(23.1) \\
(1.6) \\
(20.6) \\
(3.3) \\
(0.83)\end{array}$} & Tabaquismo, I n (\%) & 18 & $(15.0)$ \\
\hline & & & Sedentarismo & 95 & $(78.5)$ \\
\hline & & & Actividad Física ** & 26 & $(21.4)$ \\
\hline & & & Hipolipemiantes & & \\
\hline $\begin{array}{l}\text { Evento cerebrovascu } \\
\text { Isquémico } \\
\text { Hemorrágico }\end{array}$ & $\begin{array}{c}25 \\
2\end{array}$ & $\begin{array}{c}(20.6) \\
(1.6)\end{array}$ & $\begin{array}{l}\text { Estatınas } \\
\text { Fibratos } \\
\text { Ninguno }\end{array}$ & $\begin{array}{c}2 \\
49\end{array}$ & $\begin{array}{l}(1.63) \\
(40.5)\end{array}$ \\
\hline \multicolumn{6}{|c|}{$\begin{array}{l}\text { *Desviación estándar. † Estrato socioeconómico definido por Departamento Administrativo de Catastro Distrital y referido por el paciente. Es de } 1 \text { a } 6 \text {. } ¥ \text { Insuficiencia renal crónica definida } \\
\text { como depuración de creatinina menor de } 30 \mathrm{ml} / \mathrm{min} \text {. } \$ \text { TSH dato disponible para } 11 \text { pacientes. Se solicitó TSH sólo en quienes referían antecedente de hipotiroidismo y que no tenían report } \\
\text { de TSH en últimos } 3 \text { meses. II Tabaquismo definido por ATP III cualquier cigarrillo en el último mes. ** Actividad física definida como } 30 \text { minutos o más de actividad física moderada } \\
\text { todos los días o al menos } 5 \text { veces a la semana. }\end{array}$} \\
\hline
\end{tabular}

tes fueron: hipertensión arterial $102(84.3 \%)$, DM tipo 2 61 (50.4\%), enfermedad renal crónica 43 (38\%) y la ECV isquémica $25(20.6 \%)$.

El IMC promedio fue de $26.8 \mathrm{Kg} / \mathrm{m}^{2}$. Ochenta y un paciente $(66.6 \%)$ tuvieron un peso por encima de lo normal, de los cuales $21(17.3 \%)$ tenían algún grado de obesidad. La obesidad abdominal o visceral (central) fue mayor en las mujeres que en los hombres, $37(56.0 \%)$ y $19(34.5 \%)$ respectivamente. El tabaquismo estuvo presente en el $15 \%$ de los pacientes y el sedentarismo en el 78.5\% (Tabla 1).

En cuanto al tratamiento, $70(57.7 \%)$ recibían estatinas (todos ellos lovastatina), 2 (1.63\%) fibratos (gemfibrozil) y $49(40.5 \%)$ no reciben ningún hipolipemiante. Ninguno de los pacientes había suspendido el medicamento hipoli- pemiante cuando éste le había sido formulado y ninguno presentó efectos adversos derivados de las estatinas que obligara su suspensión. Las frecuencias absolutas y relativas de cumplimento de metas de colesterol LDL, HDL, no HDL y triglicéridos en los distintos grupos y según comorbilidades se discriminan en la Tabla 2. Se encontró un cumplimiento de metas de colesterol LDL en $67(55.3 \%)$ del total de pacientes estudiados, de colesterol HDL en 38 (31.4\%), de colesterol no HDL en $62(51.2 \%)$ y de triglicéridos en $63(51 \%)$. Entre los pacientes analizados, $21(17.3 \%)$ se consideraron de muy alto riesgo. De éstos, 14 (66.6\%) se encontraban en metas terapéuticas.

Se observó una mayor frecuencia de cumplimiento de metas de colesterol LDL en las mujeres 41 (62.1\%) respecto 
de los hombres 26 (47.2\%), sin embargo para el colesterol HDL y los triglicéridos el cumplimiento fue mayor en el género masculino. Hubo mejor cumplimiento de metas en todo el perfil lipídico en pacientes diabéticos comparados con los no diabéticos; sin embargo, en pacientes con antecedente de enfermedad coronaria un menor porcentaje cumplieron con las metas con relación a los que no tenían este antecedente. Situación semejante se vio en el subgrupo de pacientes con hipotiroidismo con relación a los que no tenían esta patología. Llamativamente, en el grupo que recibía estatinas previamente cumplieron con la meta 38 $(54.2 \%)$ con relación a 31 (63.2\%) en los que no las recibían (Tabla 2). Estas diferencias no fueron sujetas a análisis de significancia estadística teniendo en cuenta que algunos subgrupos contaban con pocos pacientes.

\section{Discusión}

El presente estudio es la primera investigación sobre cumplimiento de metas de lípidos según las guías ATP III en pacientes de prevención secundaria, que se realiza en el HSJ de Bogotá. Se encontró que los porcentajes de cumplimiento de metas de lípidos resultan bajos, si se tiene en cuenta el beneficio clínico demostrado y las recomendaciones dadas por las guías con respecto al cumplimiento de metas en los niveles de lípidos (6-9,16-19).

Diversos estudios han evaluado el cumplimiento de metas de lípidos en pacientes con diferentes perfiles de riesgo cardiovascular en el contexto ambulatorio. Estudios iniciales (realizados en la década de los 90) mostraron una tasa baja de cumplimiento de las metas del perfil lipídico, sin embargo, posterior al reporte del ATPIII, se ha notado cierta mejoría en el cumplimiento de estas metas. Esto es evidente en el estudio "The Lipid Treatment Assessment Project"
(LTAP), en el cual, el primer reporte publicado en el año 2000, mostró un cumplimiento de metas de colesterol LDL de $38 \%$ en todos los pacientes y de $18 \%$ en pacientes con EC (13), mientras que en el LTAP 2 el cumplimiento estuvo entre 47 y $84 \%$ según el país evaluado, $67 \%$ en el grupo de alto riesgo y entre $16-37 \%$ en el de muy alto riesgo (20).

Estudios en diversos países han mostrado tasas variables de cumplimiento de metas. El The NCEP Evaluation ProjecT Utilizing Novel E-Technology (NEPTUNE II), realizado en EU, reportó una tasa de éxito para colesterol LDL de $67 \%$ (21). Por su parte, el estudio canadiense realizado por Yan et al. reportó en pacientes diabéticos y/o con EC una tasa de cumplimiento de 51.2\%. (22). En un estudio francés se cumplió la meta en $39 \%$ de los pacientes con alto riesgo cardiovascular (23), mientras que un estudio en Grecia mostró un cumplimiento máximo de 50\% (24) y en la cohorte española del estudio DYSIS se cumplió en 36.9\% para el colesterol LDL (25). Por lo tanto, los porcentajes bajos de cumplimiento obtenidos en nuestros resultados posiblemente hacen parte de una realidad vista en otros países y que pone en evidencia que probablemente no se les está otorgando el beneficio máximo a estos pacientes de prevención secundaria.

Otro hallazgo importante evidenció que un porcentaje importante de los pacientes incluidos no recibieron tratamiento con estatinas u otros hipolipemiantes a pesar de no tener un buen cumplimiento de las metas $(6,7)$. Esta situación ha sido observada en otros estudios (22). También es importante resaltar que la única estatina formulada a los pacientes fue la lovastatina, medicamento de menor potencia frente a otras estatinas, y que hasta el momento tiene su mayor evidencia en el tratamiento de pacientes de prevención primaria (1). Esta situación es muy diferente a

Tabla 2. Cumplimiento metas de lípidos según guías ATP III.

\begin{tabular}{|c|c|c|c|c|c|c|c|c|}
\hline \multirow[b]{2}{*}{ Total $(\%)$} & \multicolumn{2}{|c|}{ LDL } & \multicolumn{2}{|c|}{ HDL } & \multicolumn{2}{|c|}{ no HDL } & \multicolumn{2}{|c|}{ TG } \\
\hline & 67 & $(55.3)$ & 38 & $(31.4)$ & 62 & $(51.2)$ & 63 & (52) \\
\hline Hombres, n (\%) & 26 & $(47.2)$ & 21 & $(38.1)$ & 25 & $(45.4)$ & 32 & $(58.1)$ \\
\hline Mujeres, n (\%) & 41 & $(62.1)$ & 17 & $(25.7)$ & 37 & $(56)$ & 31 & $(46.9)$ \\
\hline \multicolumn{9}{|l|}{ Estrato socioeconómico } \\
\hline Estrato 1 y 2 & 12 & $(38.7)$ & 3 & $(9.0)$ & 11 & $(36.3)$ & 11 & $(36.3)$ \\
\hline Estrato 3 y 4 & 39 & $(44.3)$ & 35 & $(39.9)$ & 51 & $(57.9)$ & 52 & (59) \\
\hline Con Enfermedad Coronaria & 8 & $(42.1)$ & 7 & $(36.8)$ & 4 & $(21)$ & 7 & $(36.8)$ \\
\hline Sin Enfermedad Coronaria & 68 & $(66.6)$ & 44 & $(43.1)$ & 75 & $(73.5)$ & 49 & (48) \\
\hline Diabéticos & 25 & $(40.9)$ & 38 & $(62.2)$ & 23 & $(37.7)$ & 24 & $(39.3)$ \\
\hline No Diabetes & 15 & (25) & 28 & $(46.6)$ & 15 & (25) & 14 & $(23.3)$ \\
\hline Hipotiroidismo & 9 & (36) & 7 & (28) & 12 & $(48)$ & 13 & (52) \\
\hline Sin Hipotiroidismo & 42 & $(43.75)$ & 31 & $(32.2)$ & 50 & $(52)$ & 50 & (52) \\
\hline \multicolumn{9}{|l|}{ Hipolipemiante } \\
\hline Estatina & 38 & $(54.2)$ & 24 & $(34.2)$ & 30 & $(42.8)$ & 34 & $(48.5)$ \\
\hline Ninguno & 31 & $(63.2)$ & 15 & $(30.6)$ & 32 & $(65.3)$ & 30 & $(61.2)$ \\
\hline
\end{tabular}


la evidencia que muestra estudios realizados en otros países. En España un informe reciente demostró que más de $80 \%$ de los pacientes de prevención primaria y secundaria evaluados recibieron estatinas de mayor potencia (simvastatina o atorvastatina) (25). Por su parte en el estudio canadiense, la lovastatina se prescribió a menos de $2 \%$ de la población. Una de las posibles razones para este hallazgo es que la lovastatina es la única estatina (hasta el momento de realizar el estudio) incluida en el manual de Medicamentos y Terapéutica del Sistema General de Seguridad Social en Salud colombiano, lo cual puede llegar a limitar la formulación de las otras estatinas.

Por otra parte, ninguno de los pacientes ingresados en el estudio había suspendido el medicamento hipolipemiante cuando éste le había sido formulado y ninguno presentó efectos adversos derivados de las estatinas que obligara su suspensión. Ello corresponde con reportes en la literatura mundial en los cuales el porcentaje de efectos adversos oscila entre 0.1 y $1.5 \%(12,26)$. Aunque información publicada recientemente mantiene abierta la discusión acerca de este punto $(27,28)$. Así mismo, la prevalencia de otros factores de riesgo modificables como el sobrepeso, la obesidad central y el sedentarismo se encontró en una proporción alta de los pacientes estudiados. Esto es consistente con otros reportes de nuestro medio (29).

Nuestro estudio presenta varias limitaciones. En primer lugar, no se estableció el tiempo que tenían los pacientes de estar recibiendo la lovastatina. Esto es importante, ya que se necesitan por lo menos tres meses de estar recibiendo este medicamento para que tenga efecto sobre el perfil lipídico. En segundo lugar, el estudio se realizó en pacientes hospitalizados, en los cuales el estrés agudo de la hospitalización puede modificar el perfil lipídico. Al respecto, encontramos varios grupos de trabajo que midieron el perfil lipídico en las primeras 24 horas de hospitalización en poblaciones diversas como falla cardiaca descompensada (30), enfermedad arterial coronaria $(31,32), \operatorname{ECV}(33,34)$, adultos mayores (35) y cirugía general (36). De hecho se ha recomendado la medición del perfil lipídico durante la hospitalización, en pacientes con patologías específicas como el infarto de miocardio y el ECV agudo $(14,15)$.

Si bien es cierto, se ha visto que los niveles de colesterol total y LDL pueden disminuir al ingreso del paciente, el impacto sobre los triglicéridos y el colesterol HDL son menores, y la información en cuanto a la magnitud del cambio no es consistente (37). Adicionalmente, dentro de las estrategias para desarrollar programas preventivos efectivos, se ha enfatizado en el inicio de las terapias de prevención secundaria antes del egreso hospitalario de pacientes admitidos por enfermedad cardiovascular (38), de hecho, se ha demostrado el beneficio de iniciar terapia para disminuir los niveles de lípidos durante la hospitalización inicial (39).

En tercer lugar, la medición se realizó en las primeras 24 horas, sin embargo otros factores como el estado de ayuno no fue posible controlarlo, lo cual puede ser otra fuente de sesgo de medición. Por último, el estudio fue realizado en un centro único hospitalario, lo cual limita la validez externa de sus resultados.

Como conclusión podemos decir que en la población estudiada hubo un bajo cumplimiento de metas en el perfil lipídico de colesterol LDL, no HDL y triglicéridos en casi en la mitad de los individuos, y en una mayor proporción en el colesterol HDL, según las establecidas por el ATP III, lo cual resulta preocupante si se tiene en cuenta que se requiere un cumplimiento estricto de las mismas para impactar favorablemente la morbimortalidad de estos pacientes. Se deben realizar esfuerzos integrados multidisciplinarios para mejorar el cumplimiento de metas de lípidos en pacientes de prevención secundaria atendidos en el HSJ de Bogotá.

\section{Agradecimientos}

Al grupo de residentes de medicina interna del HSJ por colaborar en el proceso de recolección de los pacientes y a la división de investigaciones de la FUCS por el valioso aporte en la realización del presente estudio.

\section{Conflicto de interés}

Los autores declaran que no tienen conflicto de intereses.

\section{Referencias}

1. Lardizabal J, Deedwania P. Lipid-lowering therapy with statins for the primary and secondary prevention of cardiovascular disease. Cardiol Clin 2011; 29: 87-103.

2. Gupta R, Deedwania P. Interventions for cardiovascular disease prevention, Cardiol Clin 2011; 29: 15-34.

3. http://www.dane.gov.co/index.php?option=com_content\&view=article\&id=63 2\&Itemid=119. Acceso 12-01-12.

4. Mendoza F., Beltrán R., Isaza D., Jaramillo C. Guías Colombianas de Cardiología Síndrome Coronario Agudo con Elevación del ST (Infarto Agudo del Miocardio con Elevación del ST). Rev Colomb Cardiol 2010; 17 (Supl.3): 121-273.

5. Opie L. Lipid- Lowering and antiatherosclerotic drugs. In: Elseviers saunders, editor. Drugs for the heart. Seven edition Ed. 2009.

6. Smith SC, Jr., Benjamin E, Bonow R, Braun L, Creager M, Franklin B, et al. AHA/ACCF Secondary prevention and risk reduction therapy for patients with coronary and other atherosclerotic vascular disease: 2011 update. Circulation 2011; 124: 2458-2473.

7. Grundy SM, Cleeman JI, Merz C, Brayan Brewer H, Clarck L, Hunninghake D, et al. Implications of recent clinical trials for the National Cholestero Education Program Adult Treatment Panel III guidelines. Circulation 2004, 110(2): 227-239.

8. Kearney PM, Blackwell L, Collins R, Keech A, Simes J, Peto R, et al. Efficacy of cholesterol-lowering therapy in 18,686 people with diabetes in 14 randomised trials of statins: a meta-analysis. Lancet 2008; 371(9607): 117-125.

9. O'Keefe JH, Carter MD, Lavie CJ. Primary and secondary prevention of cardiovascular diseases: a practical evidence-based approach. Mayo Clin Proc 2009; 84(8): 741-757.

10. Executive Summary of The Third Report of The National Cholesterol Education Program (NCEP). Expert Panel on Detection, Evaluation, And Treatment of High Blood Cholesterol In Adults (Adult Treatment Panel III). JAMA 2001; 285(19): 2486-2497.

11. Guidelines for the management of dyslipidaemias. European Heart Journal 2011; 32: 1769-1818.

12. Jones P, Kafonek S, Laurora I, Hunninghake D. Comparative dose efficacy study of atorvastatin versus simvastatin, pravastatin, lovastatin, and fluvastatin in patients with hypercholesterolemia (the CURVES study). Am J Cardiol 1998; 81(5): 582-587.

13. Pearson TA, Laurora I, Chu $\mathbf{H}$, Kafonek $\mathbf{S}$. The lipid treatment assessment project (L-TAP): a multicenter survey to evaluate the percentages of dyslipidemic patients receiving lipid-lowering therapy and achieving low-density lipoprotein cholesterol goals. Arch Intern Med 2000; 160(4): 459-467.

14. Antman EM, Anbe DT, Armstrong PW, Bates E, Green L, Hand M, et al. ACC/AHA guidelines for the management of patients with ST-elevation 
myocardial infarction--executive summary: a report of the American College of Cardiology/American Heart Association Task Force on Practice Guidelines (Writing Committee to Revise the 1999 Guidelines for the Management of Patients With Acute Myocardial Infarction). Circulation 2004; 110(5): 588-636.

15. Adams HP, Del Zoppo G, Alberts MJ, Bhatt D, Brass L, Furlan A, et al. Guideline for early managmet of adults with ischemic Stroke. Stroke 2007; 38: $1655-1711$

16. K/DOQI clinical practice guidelines for chronic kidney disease: evaluation, classification, and stratification. Am J Kidney Dis 2002; 39(2 Suppl 1): S1-266.

17. World healt organization.obesity: prevention and managning the global epidemic, Geneva, Switzerland: world healt organization; 2000. WHO Technical Report series.

18. The 4S Investigators. Randomised trial of cholesterol lowering in 4444 patients with coronary heart disease: the Scandinavian Simvastatin Survival Study (4S). Lancet 1994; 344(8934): 1383-1389.

19. Colhoun HM, Betteridge DJ, Durrington PN, Hitman G, W Neil HA, Livingstone SJ, et al. Primary prevention of cardiovascular disease with atorvastatin in type 2 diabetes in the Collaborative Atorvastatin Diabetes Study (CARDS): multicentre randomised placebo-controlled trial. Lancet 2004; 364(9435): 685-696.

20. Waters DD, Brotons C, Chiang CW, Ferrieres J, Foody J, Jukema W, et al. Lipid treatment assessment project 2: a multinational survey to evaluate the proportion of patients achieving low-density lipoprotein cholesterol goals. Circulation 2009; 120(1): 28-34.

21. Davidson M, Maki K, Pearson T, Pasternak R, Deedwania P, McKenney J, et al. Result of the National Cholesterol Education (NCEP) Program evaluation project utilizing novel E-technology (NEPTUNE) II survey and implications for treatment under the recent NCEP writing group recommendations. Am J Cardiol 2005; 96: 556-563.

22. Yan A, Yan R, Tan M, Hackman D, Leblanc K, Kertland H, et al. Contemporary management of dyslipidemia in high-risk patients: targets still not met. Am J Med 2006; 119: 676-683.

23. Ferrieres J, Berard E, Crisan O, Bongard V. Residual dyslipidaemia after statin treatment in France: Prevalence and risk distribution. Arch Cardiovasc Dis 2010; 103(5): 302-309.

24. Elisaf MS, Nikas N. Centralized Pan-European survey on the undertreatment of hypercholesterolemia in patients using lipid lowering drugs--the CEPHEUSGreece survey. Angiology 2010; 61(5): 465-474.

25. Gonzalez-juanatey J, Millan J, Alegria E, Guijarro C, Lozano J, Vitale G. Prevalencia y caracteristicas de la dislipidemia en prevencion primaria y secundaria tratados con estatinas en España. Estudio DYSYS-ESPAÑA. Rev Esp Cardiol 2011; 64(4): 286-294.
26. Bradford RH, Downton M, Chremos AN, Langendorfer A, Stinnett S, Nash DT, et al. Efficacy and tolerability of lovastatin in 3390 women with moderate hypercholesterolemia. Ann Intern Med 1993; 118(11): 850-855.

27. Fernández G, Spatz ES, Jablecki C, Phillips PS. Statin myopathy: a common dilemma not reflected in clinical trials. Cleve Clin J Med 2011; 78: 393-403.

28. Maningat $\mathbf{P}$, Breslow J. Needed: Pragmatic Clinical Trials for Statin- Intolerant Patients. N Eng J Med 2011; 365 (24): 2250-2251.

29. Lombo B, Satizabal C, Villalobos C, Tique C, Kattah W. Prevalencia del síndrome metabólico en pacientes diabéticos. Acta Med Colomb 2007; 32(1): 9-15.

30. Horwich T, Hernandez A, Dai D, Yancy C, Fonarrow G. Cholesterol levels and in-hospitl mortality in patients with acute descompensated heart failure. Am Heart J 2008; 156: 1170-6.

31. Harats D, Leibovitz E, Maislos M, Wolfovitz E, Chajek-Shaul T, Leistesdorf E, et al. Cardiovascular risk assessment and treatment to target low density lipoprotein levels in hospitalized ischemic heart disease patients: results of the HOLEM study. IMAJ 2005; 7: 355-359.

32. Albert N, Birtcher K, Cannon C, Goff D, Mulgund J, Liang L, Fonarrow G. Factors associated with discharge lipid-lowering drugs prescription in patients hospitalized for coronary artery disease (from the get with the guidelines database). Am J Cardiol 2008; 101: 1242-1246.

33. Smith E, Abdullah A, Amirfarzan H, Schwamm L. Serum lipid profile on admission for ischemic stroke. Neurology 2007; 68: 660-665.

34. Smith E, Pan W, Olson D, Reeves M, Ovbiagele B, Peterson E, et al. Frecuency and determinants of lipid testing in ischemic stroke and transient ischemic attack. Findings from get with the gudelines-stroke. Stroke 2010; 41: 232-238.

35. Onder G, Landi F, Volpato S, Fellin R, Carbonin P, Gambassi G, Bernabei R. Serum cholesterol levels and in-hospitalized mortality in the elderly. Am J Med 2003; 115(4): 265-271

36. Delgado-Rodriguez M, Medicna-Cuadros M, Gomez-Ortega A, MartínezGallego G, Mariscal-Ortiz M, Martínez-Gonzales M, Sillero-Arenas M. Cholesterol and serum albumin levels as predictors of cross infection, death, and length of hospital stay. Br J Surg 2007; 94(3): 369-75.

37. Nawaz H, Patton B, Yanchou V, Dhond A, Plavec M, Katz D. Repeated serum lipid measurements during the peri-hospitalization period. Am J Cardiol 2006; 98: 1379-1382.

38. McKinnon PS, Seaton T, Noirot LA, Reichley RM, Heard KM, Shannon WD, et al. Improving adherence to dyslipidemia medication guidelines in hospitalized diabetic patients using a technology-assisted pharmacist. Annu Symp Proc 2008: 868.

39. Steinhoff J, Smith S. Putting prevention into daily practice. Cardiol Clin 2003 21: $471-482$ 